Calibration and Characterization of Single Photon Counting Cameras for Short-Pulse Laser Experiments

B. R. Maddox, H.-S. Park, B. A. Remington, M. McKernan

May 8, 2008

17th Topical Conference on High-Temperature Plasma Diagnostics

Albuquerque, NM, United States

May 11, 2008 through May 15, 2008 
This document was prepared as an account of work sponsored by an agency of the United States government. Neither the United States government nor Lawrence Livermore National Security, LLC, nor any of their employees makes any warranty, expressed or implied, or assumes any legal liability or responsibility for the accuracy, completeness, or usefulness of any information, apparatus, product, or process disclosed, or represents that its use would not infringe privately owned rights. Reference herein to any specific commercial product, process, or service by trade name, trademark, manufacturer, or otherwise does not necessarily constitute or imply its endorsement, recommendation, or favoring by the United States government or Lawrence Livermore National Security, LLC. The views and opinions of authors expressed herein do not necessarily state or reflect those of the United States government or Lawrence Livermore National Security, LLC, and shall not be used for advertising or product endorsement purposes. 


\title{
Calibration and Characterization of Single Photon Counting Cameras for Short-Pulse Laser Experiments
}

\author{
B. R. Maddox, H. S. Park, B. A. Remington, and M. McKernan \\ Lawrence Livermore National Laboratory, Livermore, California 94551
}

(Dated: May 2, 2008)

\begin{abstract}
The photon counting efficiency of various CCD based cameras was studied as a function of xray energy and exposure. A pair of Spectral Instruments Model 800 CCD cameras fitted with 16 $\mu m$ thick back-illuminated CCDs were calibrated at low x-ray energy using two well established histogram methods, a standard pixel for pixel histogram and the single pixel event histogram method. In addition, two new thick substrate CCDs were evaluated for use at high energy. One was a commercially available Princeton Instruments LCX1300 deep depletion CCD camera while the other was a custom designed $650 \mu \mathrm{m}$ thick partially depleted CCD fitted to a SI 800 camera body. It is shown that at high x-ray energy, only a pixel-summing algorithm was able to derive spectral data due to the spreading of x-ray events over many pixels in the thicker substrate CCDs. This paper will describe the different algorithms used to extract spectra and the absolute detection efficiencies using these algorithms. These detectors will be very useful to detect high-energy x-ray photons from high-intensity short pulse laser interactions.
\end{abstract}




\section{INTRODUCTION}

Single photon counting using CCD cameras offers a convenient method of providing a measure of the x-ray production efficiency of short pulse laser targets, such as K $\alpha$ and Compton $\mathrm{x}$-ray backlighters, in laser plasma experiments ${ }^{1,2}$. Quantitative analysis of the x-ray production is much more straightforward in these devices, compared to crystal spectrometers and other energy dispersive devices which require detailed knowledge of the dispersive element dispersion and efficiency as well as the detector response as a function of energy. In contrast, a CCD responds linearly with x-ray energy and the data analysis of the resulting CCD images is relatively simple and uses histograms to reconstruct the x-ray spectrum.

However, the CCD cameras commonly used today for single photon counting are limited to a maximum x-ray energy of around $22 \mathrm{keV}$ due to thin Si substrates. A thicker Si substrate is required for efficient detection of x-ray energies in excess of $22 \mathrm{keV}$. Another important limitation of single photon counting using CCDs is that it is often times difficult to ensure that the x-ray exposures obtained using CCD cameras stays within the single

photon counting regime of 1 detected x-ray event per 100 pixels REFERENCE. This could be due to an widely unknown production efficiency or large amount of x-ray background.

In this study, two CCD cameras commonly used for single photon counting spectroscopy were calibrated at low energy. Their response using two different image analysis methods are presented and their efficiency as a function of exposure level and histogram method is investigated. This information is important for correcting single photon counting CCD data that has been exposed over the single photon counting threshold. Two thick substrate cameras have also been evaluated for use at high x-ray energy and a new image analysis method is presented which allows spectral data to be reconstructed from these thick substrate chips.

\section{EXPERIMENTAL SETUP}

At low x-ray energy, a pair of Spectral Instruments Model 800 CCD cameras (SI800-104 and SI800-116) fitted with 2048x2048 (13.5 $\mu \mathrm{m}$ pixel size) $16 \mu \mathrm{m}$ thick back-illuminated EEV CCDs were calibrated at 5.899 and $22.162 \mathrm{keV}$ using ${ }^{55} \mathrm{Fe}$ and ${ }^{109} \mathrm{Cd}$ sealed sources, respectively. Both cameras were fitted with $500 \mu m$ thick Be windows, to prevent exposure 
to ambient light, and cooled to $-40^{\circ} \mathrm{C}$ to minimize the dark current. The activity of each source was calibrated by measuring the main x-ray emission lines for each source, Ag and $\mathrm{Mn} \mathrm{K} \alpha$ and $\mathrm{K} \beta$ for ${ }^{55} \mathrm{Fe}$ and ${ }^{109} \mathrm{Cd}$ respectively, using an AmpTek XR100-CR Si detector. Coupled with an AmpTek MCA8000A multichannel analyzer, we were able to measure the activities of our sources to be $0.59 \mathrm{mCi}$ and $0.30 \mathrm{mCi}$ for $55 \mathrm{Fe}$ and Cd109 with an error of $+/-10 \%$, estimated by comparing our measured values with the factory specified values.

A series of exposures of varying dose were performed on SI800-104 using both ${ }^{109} \mathrm{Cd} 109$ and ${ }^{55} \mathrm{Fe}$. Each sealed source was held in a lead housing and was hand shuttered using a 4 mm thick lead curtain. The doses were varied between 0.1 and 10 times the ideal single hit exposure level by varying the integration time and the distance between the source and the CCD plane. The use of hand-shuttering introduced a 2 second error in the integration time. Combined with the $10 \%$ error in source calibration a conservative estimate of the error in our dose calculations, and hence our efficiency measurements, is $\pm 15 \%$.

At high x-ray energy, we exposed two thick substrate cameras to an ${ }^{241} \mathrm{Am}$ sealed source which, among other lines, has a gamma emission at $59.54 \mathrm{keV} \mathrm{keV}^{3}$. The ratio of this line to the other, lower energy emissions was sufficient to allow a measurable exposure at 59.54 $\mathrm{keV}$ without exceeding the single hit exposure level with the other, lower energy emissions. The first camera evaluated was a commercially available PI-LCX1300 camera by Princeton Instruments which uses a deep depletion 1340x1300 CCD chip with an effective thickness of $50 \mu \mathrm{m}$. Deep depletion CCD chips use a higher resistivity, epitaxial Si layer to limit electron diffusion in the active region without needing high bias voltages, allowing thicker active regions. The second camera tested used a custom designed $650 \mu \mathrm{m}$ thick CCD developed as part of the SNAP CCD project at Lawrence Berkeley National Lab fitted to an SI800 camera body ${ }^{4}$. The exposure on both cameras was adjusted so that they were operating in the single hit regime and used the same exposure setup described above for the pair of commercial SI800 cameras.

\section{IMAGE ANALYSIS}

Figure 1(inset) shows a cropping of a typical CCD image obtained using ${ }^{109} \mathrm{Cd}$ at the ideal single hit exposure level and shows 3 distinct types of events. The first (labeled Type

1) occurs when the entire charge cloud generated in the Si substrate during an x-ray event 
is captured in a single pixel well. In this type of event, the pixel intensity is proportional to the x-ray energy and can be written as $I_{p i x e l}=E_{p p} \times$ Gain where $E_{p p}$ is the pair production energy and Gain is the camera gain. At $-40^{\circ} E_{p p}=3.72 \mathrm{eV} / \mathrm{e}-\mathrm{h}$ pair ${ }^{5}$. The values for Gain differ for each camera and were specified by the factory. The second type of event (labeled Type 2) occurs over 2 or 3 pixels. These events arise when the x-ray interacts close to the boundary of a pixel. Then there are those split events which form fuzzy patches in the image (Type 3). These occur when an x-ray interacts in a field-free region of the CCD. In this case, the electron cloud generated during the event is allowed to spread to multiple pixels before being collected. In these latter two even types, it is the summed pixel value which is proportional to the x-ray energy.

The CCD images obtained at low x-ray energy on SI800-104 and SI800-116 were analyzed using two different histogram methods, a standard histogram and a "single pixel event" histogram method which attempts to only count Type 1 events. In both methods, a dark image is subtracted before any histograms are performed. The resulting spectra, applied to the image shown in Fig. 1(inset) are shown in Fig. 1. The broad, sloping background seen using the standard histogram method is a result of the large number of Type 3 pixel events. This broad, non-physical background needs to be subtracted from the spectrum before analysis of the detection efficiency can occur. The "single pixel event" only adds a pixel to the histogram if $98 \%$ of the energy in a 3x3 block centered around that pixel is in the center pixel ${ }^{6}$. Figure 1 also shows the "single event" histogram method applied to the same CCD image illustrating the dramatic disappearance of the broad low energy background produced by the "standard" histogram method. This "single pixel event" spectrum can then be directly integrated to measure the detection efficiency.

\section{RESULTS AND DISCUSSION}

The detection efficiency, $\epsilon_{e f f}(E)$, for a given camera/histogram combination is defined as the number of histogram counts in the main emission line divided by the total number of incident x-rays at that energy. Because we are summing only the counts surrounding the main emission line, this definition of detection efficiency is a function of not only the absorption probability in the Si layer, but also the probability that an x-ray will deposit all its energy in the Si via the photoelectric effect and that the resulting charge cloud will 


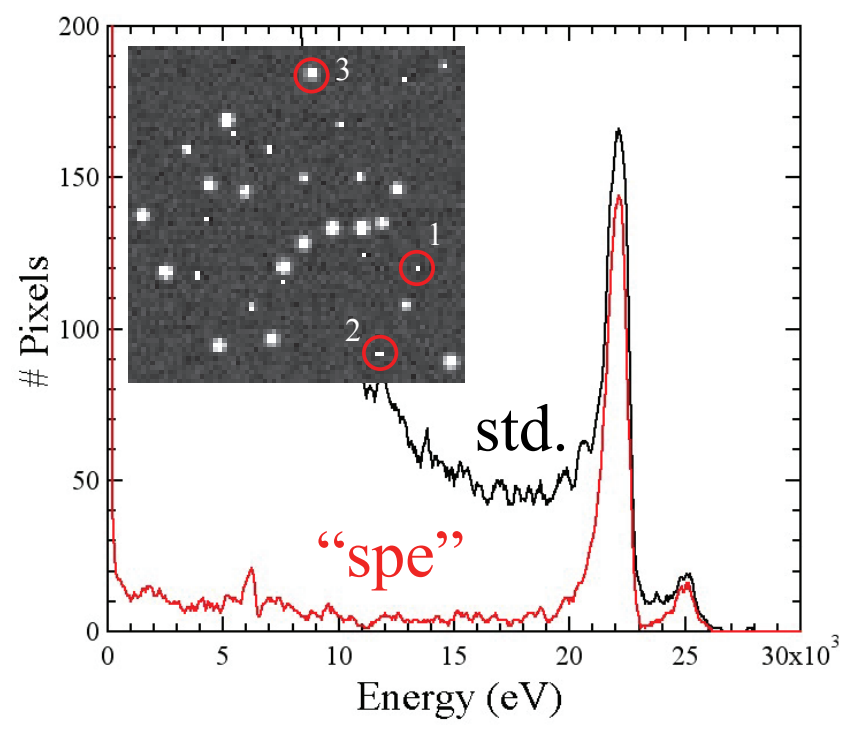

FIG. 1: Standard and "single pixel event" histogram results from an ideal single hit exposure using ${ }^{109} \mathrm{Cd}$. (insert) Image crop showing the 3 types of photon events: (1) single pixel events (2) split pixel events where the x-ray interacted near a pixel boundary (3) blobs where the x-ray interacted in a field-free region producing a fuzzy spot. (b) standard and "single pixel event" histograms of the full CCD image shown in (a). A broad sloping background is seen in the standard histogram due to the split and blob x-ray events.

be collected in a single pixel well, all of which are a function of x-ray energy. This can be written as $\epsilon_{e f f}(E)=\epsilon_{S i}(E) \times \epsilon_{P E F}(E) \times \epsilon_{S H}(E)$ where $\epsilon_{S i}(E)$ is the absorption probability in the Si layer, $\epsilon_{P E F}(E)$ is the photoelectric fraction in $\mathrm{Si}$, and $\epsilon_{S H}(E)$ is the probability that the charge cloud will be collected in a single pixel ${ }^{1}$. The number of x-ray photons incident at the main emission energy of each source can be calculated using

$$
\# \gamma^{\prime} s=\beta \times t \times \frac{\Omega}{4 \pi} \times \sum_{E} I(E) T(E)
$$

where $\beta$ is the activity in dps, $t$ is the integration time, $\Omega$ is the solid angle subtended by the CCD chip, and $I(E)$ and $T(E)$ are the decay probability and transmission through our apparatus, respectively. For ${ }^{55} \mathrm{Fe}$ the transmission term is the transmission through air and the $500 \mu \mathrm{m}$ Be window. Our ${ }^{109} \mathrm{Cd}$ source was encased in an acrylic disk so $\mathrm{T}(\mathrm{E})$ includes an additional term corresponding to the transmission through $2.77 \mathrm{~mm}$ of plastic. We used dry air at STP to calculate the absorption through air for both sources.

Figure 2(a) shows our efficiency results at $5.899 \mathrm{keV}$ as a function of CCD exposure level 

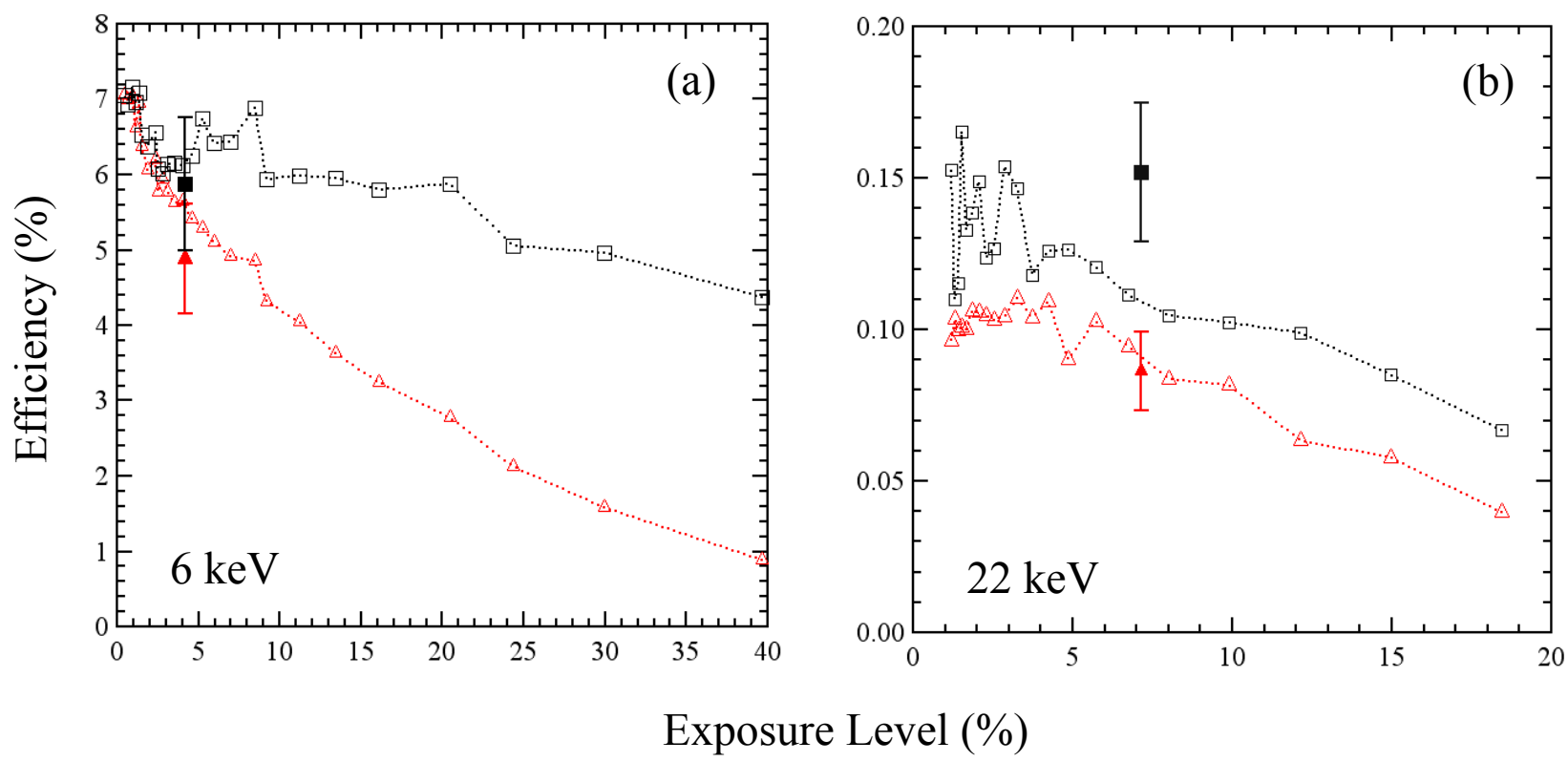

FIG. 2: Efficiency results at $5.899 \mathrm{keV}$ (a) and $22.162 \mathrm{keV}$ (b) for SI800-104 (open) and Si116 (solid) using the standard histogram method (squares) and the "single pixel event" method (triangles). The dashed horizontal lines show the average efficiency derived from the data points between 0 and $5 \%$ exposure level at $5.899 \mathrm{keV}$ and between 0 and $7 \%$ exposure level at $22.162 \mathrm{keV}$.

using both the standard and "single pixel event" histogram methods. The exposure level is defined as the number of pixels greater than $3 \times$ the background HWHM divided by the total number of pixels. Since the standard histogram method counts each pixel individually, at low exposure level we expect the calculated efficiency to be the same as that derived using the "single event" method. Indeed Fig. 2(a) shows that the detection efficiency is the same for both histogram methods up to an exposure level of $3 \%$. Above this exposure level the efficiency using the "single event" method continues to fall. This is due to event "pileup" where x-ray events begin to overlap. In contrast, the efficiency derived using the standard histogram method stays relatively flat, dropping by only $25 \%$ at an exposure level of $40 \%$.

Our results at $22.162 \mathrm{keV}$ are shown in Fig. 2(a) with the same $\pm 15 \%$ error bars on the SI116 results. A different story emerges between the standard and "single pixel event" histogram methods. Both methods produce a relatively constant value for the detection efficiency at low exposure levels, but are offset from each other by $25 \%$. Additionally, the efficiency using both methods seems to diminish at a similar rate. This behavior is due to the higher fraction of spread pixel events at higher x-ray energy and is also reflected by the 
much higher exposure level at the calculated ideal single hit dose compared to our results at $6 \mathrm{keV}$.

Both Fig. 2(a) and (b) show that above exposure levels of $10 \%$ the detection efficiency using the "single pixel event" method drops below the ideal exposure efficiency by more than our estimated error of $15 \%$. Thus, for exposures greater than this one cannot use a single value for the detector efficiency but must correct for CCD pileup using Figs. 2(a) and (b). The standard histogram method fares a bit better and at $5.899 \mathrm{keV}$, the efficiency stays within our $15 \%$ error estimate all the way to an exposure level of $20 \%$. Even at the an exposure level of $40 \%$ the efficiency has only dropped by $2 \times$ our error estimate. At higher x-ray energy the standard method still fails above the $10 \%$ exposure level and requires correction for CCD pileup. Therefore, we define the ideal exposure efficiency as the average efficiency between $0 \%$ and $5 \%$ total exposure. At $5.899 \mathrm{keV} e_{e f f}=6.53 \%$ and $6.24 \%$ using the standard and "single pixel event" histogram methods, respectively. At $22.162 \mathrm{keV}$ these values are $0.134 \%$ and $0.103 \%$.

These efficiency results can be extrapolated to intermediate x-ray energies by using the definition of the detection efficiency and scaling by the absorption through the $16 \mu \mathrm{mi}$ layer and the photoelectric fraction. Figure 3 shows this extrapolation out to $30 \mathrm{keV}$ from the $5.899 \mathrm{keV}$ data point, assuming that the single hit probability is constant throughout this range. The extrapolated efficiency curve is in good agreement with the $22.162 \mathrm{keV}$ data point giving a value of $0.160 \%$ at $22.162 \mathrm{keV}$ compared to the experimental value of $0.134 \%$. This difference is just outside of our experimental error estimate of $15 \%$ and is most likely due to a lower $\epsilon_{S H}$ at higher x-ray energy.

Figure 4 shows the test results for both thick substrate cameras using the two histogram methods previously describe. The standard histogram method was unable to reconstruct the $59.54 \mathrm{keV}{ }^{241}$ Am x-ray peak in either camera indicating that there were no single pixel events for either camera, most likely resulting from a combination of thick substrate and non-fully depleted operation. The "single pixel event" histogram fails to extract any sort of spectrum from either camera, consistent the results obtained through the standard histogram method.

Visual inspection of the images obtained during these ${ }^{241} \mathrm{Am}$ exposures revealed event sizes ranging from 2 pixels to events spanning a $7 \times 7$ block size. In order resolve the 59.54 keV gamma emission line a new blob summing algorithm was implemented that can sum 


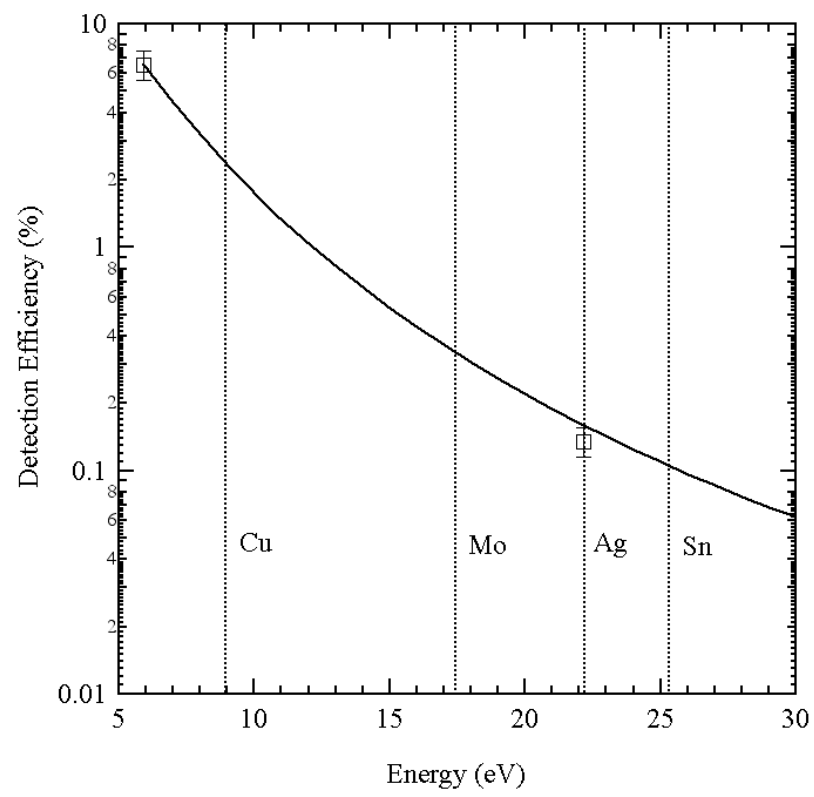

FIG. 3: Extrapolation of the efficiency results at $5.899 \mathrm{keV}$ to $30 \mathrm{keV}$ (solid line). Open squares show the experimental data. Dotted vertical lines show the $\mathrm{K} \alpha$ energies of various common laser targets.

arbitrarily sized and shaped events by summing connected pixels. A connected pixel is defined as a pixel whose ADU value is greater than $3 \times$ the background HWHM peak generated from a dark exposure. This type of algorithm is superior to a simple $n \times n$ block summing algorithm which results in severely degraded energy resolution due to multiple events smaller than the $\mathrm{n} \times \mathrm{n}$ block size occurring within a single block. Attempting to ensure proper isolation of an event contained within the $\mathrm{n} \times \mathrm{n}$ block reduces the detection efficiency, similar to our results at low energy using the "single pixel event" histogram method.

The results of the blob summing algorithm applied to the ${ }^{241} \mathrm{Am}$ using the custom CCD are also shown in Fig. 4. This algorithm was able to resolve the $59.54 \mathrm{keV}$ gamma emission peak in both cameras. However, the maximum number of connected pixels necessary to reconstruct the spectrum was different for the two cameras. The commercially available PI-LCX1300 camera only needed a maximum of 5 connected pixels to reconstruct the 59.54 kev peak whereas the custom designed CCD camera needed up to 50 connected pixels. The much larger blob size required for the custom camera is consistent with the standard histogram results which show counts in the PI-LCX up to $55 \mathrm{keV}$ compared to the custom camera which does not show any hits above $30 \mathrm{keV}$. Our custom CCD has a much thicker, 


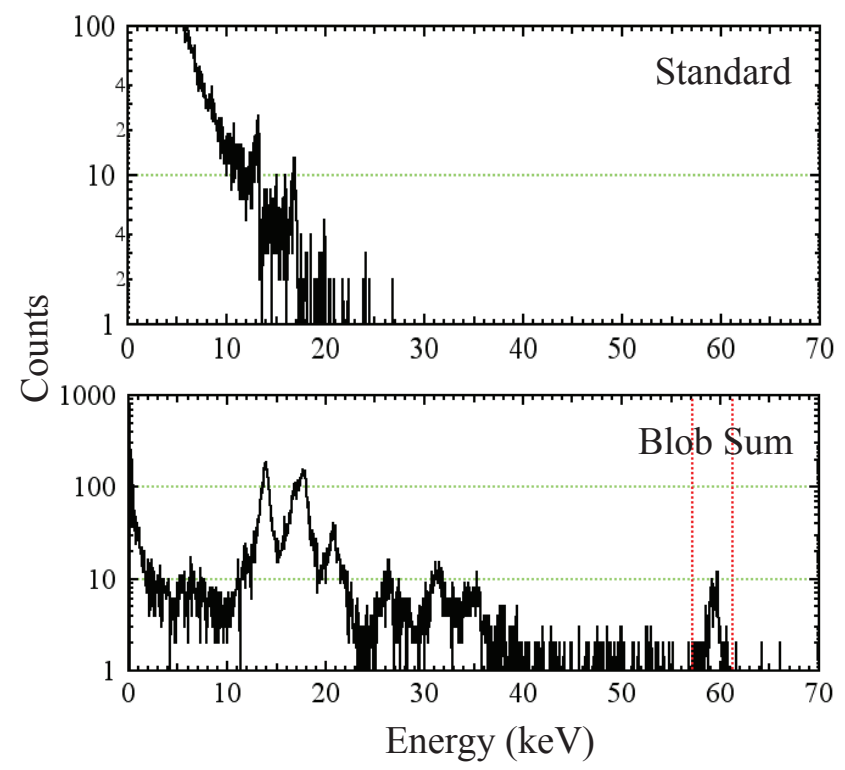

FIG. 4: Results of ${ }^{241} \mathrm{Am}$ exposures with the custom $650 \mu \mathrm{m}$ thick CCD using two different histogram methods. Only the blob summing algorithm which sums arbitrarily shaped connected pixel regions was able to reconstruct the $59 \mathrm{keV}$ peak using a maximum of 50 connected pixels on the custom SI800 camera. Similar results were obtained using the PI-LCX1300 camera with only 5 connected pixels.

non-fully-depleted substrate meaning that a charge cloud generated in the field-free region of the CCD has to travel much farther in order to be collected, and thus is allowed to spread to a larger diameter ${ }^{7}$. This, coupled with a smaller pixel size compared to the commercially available PI camera results in much larger event sizes. The efficiency at $59.54 \mathrm{keV}$ for the two thick substrate cameras was estimated to be $0.036 \%$ and $0.34 \%$ for the PI-LCX and custom camera, respectively. The order of magnitude difference in detection efficiency is due to the much thicker $650 \mu \mathrm{m}$ substrate of our custom CCD.

It should be noted that we are using a slightly different definition of detection efficiency because the histogram method used for these two thick substrate cameras attempts to count not only single pixel events, but also the spread pixel events. The detection efficiency can thus be written as $\epsilon_{e f f}=\epsilon_{S i}(E) \times \epsilon_{P E F}(E)$. Using the mean free path of a $59.54 \mathrm{keV}$ photon through Si of $13.55 \mathrm{~mm}$ and the photoelectric fraction $\epsilon_{P E F}(E=60 \mathrm{keV})=0.408 \%{ }^{8}$, we calculate the expected overall detection efficiencies of $1.89 \%$ and $0.15 \%$ for the $650 \mu \mathrm{m}$ and $50 \mu m$ substrate CCDs, respectively. These rough estimates of the expected detection 
efficiencies assume the blob summing algorithm is $100 \%$ efficient at detecting $\mathrm{x}$-ray events. Since our estimates are $5 \times$ larger than our experimental results, this implies our blob summing algorithm is only $20 \%$ efficient at properly detecting and summing spread pixel events. This could be improved by reducing the exposure further to ensure we are in the ideal single hit exposure regime.

\section{CONCLUSION}

The single photon counting detection efficiency of two SI800 cameras was measured at $5.899 \mathrm{keV}$ and $22.162 \mathrm{keV}$ using calibrated sealed sources as a function of exposure level using two histogram methods. At $5.899 \mathrm{keV}$ and the ideal single hit exposure level, the detection efficiency is $6.24 \%$ and $6.53 \%$ using the standard and "single pixel event" histogram methods with an estimated error of $\pm 15 \%$. Above $10 \%$ exposure level the "single pixel event" method deviates by more than our estimated error and requires correction for CCD pileup. The standard histogram method stays within our error estimate all the way to $25 \%$ exposure and only deviates by $30 \%$ at $40 \%$ exposure. At $22.162 \mathrm{keV}$ both histogram methods deviate by more than $15 \%$ above the $10 \%$ exposure level due to the increased number of spread pixel events at high x-ray energy. The efficiency results for camera SI800-116 are within our estimated error of those obtained on SI104.

Two thick-substrate CCD cameras were also evaluated for use as single photon counting spectrometers at high x-ray energy. A blob summing algorithm was implemented to deal with the lack of single pixel events caused by the higher x-ray energy and thicker, non fully

depleted substrates. This algorithm was able to resolve the $59.541 \mathrm{keV}$ peak of ${ }^{241} \mathrm{Am}$ using a maximum of 5 connected pixels on the PI-LCX1300 camera and 50 connected pixels on the custom $650 \mu \mathrm{m}$ thick CCD. The detection efficiencies at $59.54 \mathrm{keV}$ using the blob summing algorithm are 0.036 and $0.34 \%$ for the PI-LCX and our custom camera, respectively. This work performed under the auspices of the U.S. Department of Energy by Lawrence Livermore National Laboratory under Contract DE-AC52-07NA27344.

1 H.-S. Park, D. M. Chambers, H.-K. Chung, R. J. Clarke, R. Eagleton, E. Giraldez, T. Goldsack, R. Heathcote, N. Izumi, M. H. Key, et al., Phys. Plasmas 13, 056309 (2006). 
2 R. Tommasini, H.-S. Park, P. Patel, B. Maddox, S. L. Pape, S. P. Hatchett, B. A. Remington, M. H. Key, N. Izumi, M. Tabak, et al., AIP Conf. Proc. 926, 248 (2007).

3 Y. A. Akovali, NDS 72, 191 (1994).

4 R. J. Stover, M. Wei, K. Ji, W. E. Brown, D. K. Gilmore, S. E. Holland, D. E. Groom, M. E. Levi, N. Palaio, and S. Perlmutter, Proc. 4th ESO Workshop on Optical Detectors for Astronomy pp. 13-16 (1999).

5 C. A. Klein, J. Appl. Phys. 39, 2029 (1968).

6 F. N. Beg, A. R. Bell, A. E. Dangor, C. N. Danson, A. P. Fews, M. E. Glinsky, B. A. Hammel, P. Lee, P. A. Norreys, and M. Tatarakis, Phys. Plasmas 4, 447 (1997).

7 D. E. Groom, P. H. Eberhard, S. E. Holland, M. E. Levi, N. P. Palaio, S. Perlmutter, R. J. Stover, and M. Wei, Proc. 4th ESO Workshop on Optical Detectors for Astronomy pp. 13-16 (1999).

8 W. H. McMaster, N. K. D. Grande, J. H. Mallett, and J. H. Hubbell, Tech. Rep., Lawrence Livermore National Laboratory (1969). 